\title{
Schools and Mental Health: How Schools Support Mental Health during Pandemic-Related Disruptions
}

\author{
Dana Lev* \\ University of Bucharest, Israel
}

*Corresponding author: Dana Lev, University of Bucharest UNIBUC, 18 Ha'Sharon St. Apt 9 , Israel, Tel: +972523688772; Email: danale31@gmail.com

\section{Mini Review \\ Volume 5 Issue 2}

Received Date: August 09, 2021

Published Date: August 31, 2021

DOI: $10.23880 / \mathrm{mhrij}-16000149$

\section{Abstract}

This review addresses the emerging field which analyzes the social and emotional effects of COVID-19 related disruptions on children and adolescents. Educators and counselors, as positive influencers on a student population, have an outsized role in supporting the mental health and wellbeing of students. This review looks at school-based services which provide a platform for effective emotional and mental health support. Public health policymakers should include school systems and the support services they can provide as part of the national COVID-19 recovery plan. One specific group of students which faces particular mental health challenges is the international 'nomad' student population, popularly referred to as 'Third Culture Kids' (TCK). International schools who cater to the needs of this population must adopt an extensive integration plan for newcomers in light of the challenges presented by COVID-19 related disruptions.

Keywords: Mental Health; School System; International Education; Third Culture Kids

Abbreviations: TCK: Third Culture Kids; DL: Distance Learning; Covid-19: Coronavirus Disease 2019.

\section{Introduction}

The global outbreak of COVID-19 had a massive impact on students-primary, secondary, university, and international. Around the world, schools ceased in-person instruction and closed campuses. The consequences were colossal and affected all aspects of life, precipitating emotional trauma and economic stability. Schools were particularly affected in terms of loss of student services, lack of appropriate teaching methods, and the loosening of interpersonal relationships. Not only educational, but also health-related resources were disconnected from approximately 60 million American students [1]. Students could no longer interact on an intimate basis with teachers or peers, to service educational or emotional needs. The delivery of formal education shifted in an immediate and extreme way.

Both students and teachers were forced to adjust to new ways of teaching and learning, new ways of communicating, in particular online and via screens [2], also known as "Distance Learning" (DL). The daily pedagogical use of online communication platforms: Zoom, Teams, GoogleMeet, and Skype among others, will most likely continue until the pandemic passes [3]. This forced adjustment had a stark (almost exclusively negative) impact, not only on an academic, but on an emotional front as well as on health and general wellbeing. Taking the current data, as well as personal experience into account, this review explores the impact of pandemic-related disruptions on student mental health and proposes potential educational support solutions. 


\section{Mental Health \& Human Resilience International Journal}

\section{Student Mental Health and Wellbeing}

The term 'wellbeing' was employed by the World Health Organization (WHO) [4] in an early definition of health as "...a state of complete physical, mental, and social wellbeing and not merely the absence of disease or infirmity" (WHO). "The term wellbeing generally alludes to a range of factors in one's life that contributes towards fulfillment and good physical health" [2]. This term has become particularly relevant in discussions of the negative impacts of COVID-19 related disruptions on children and young adults. Equally relevant to the below discussion is the proposition that $50 \%$ of adult mental health problems can be identified as having onset during youth, according to Kelly's research [5].

A mental health study carried out among a university student population identified a positive connection between feelings of depression, anxiety, and stress, and states of loneliness and disconnection [6]. In a similar, inverse vein, Lyubomirsky S, et al. [7] found a correlation between feelings of happiness and positive affect to one's perception of success in work, love, health, and general feeling of being successful in life. The negative feelings and states of being described by Richardson T, et al. [6] were already on the rise well before the global crisis of 2020. Prior to the onset of the COVID-19 pandemic, Kelly H [5] reviewed worldwide studies which identified a growth in mental health problems within the younger aged population. Her research already revealed an escalating number of mental illnesses detectable in adolescents. Kelly H [5] reports that in 2017, almost $13 \%$ of the 5-19-year-olds in the UK were diagnosed with a mental health condition, in 2019, one in five Canadian youth were likewise diagnosed, and in 2016, one in six of the 6-17-yearolds in the USA were also diagnosed with a mental health condition. She highlights that $20 \%$ of adolescents globally were reportedly diagnosed with a mental health condition in 2018. Thus COVID-19 emerged against a background of preexisting mental health challenges in the youth population.

\section{COVID-19 Disruptions affect Mental Health}

The entire global population felt the effects of COVID-19 related disruptions, but several researchers have tried to identify and highlight particularly vulnerable population groups: those that risk the greatest potential negative effects of pandemic-related social restrictions. During a process of research analysis focused on the mental health outcomes of the COVID-19 global health crisis, Rajkumar RP [8] detected a common phenomenon that arose as a response: mental health problems with subsyndromal preforms. He noted vulnerable populations as those which would suffer in particular from such conditions. He presented different population groups that were identified as more vulnerable to the negative mental health effects associated with
COVID-19 related disruptions: Fakari FR, et al. [9] wrote about the vulnerability of pregnant women; Tsai J, et al. looked at homeless people [10]; Yang Y, et al. [11] examined older adults; and Yao $\mathrm{H}$, et al. [12] wrote about the mentally ill. Masonbrink AR, et al. [1] showed that children living in poverty were an extremely vulnerable group. Likewise, Kelly H [13] conveyed that teachers and school leadership were another population group that is vulnerable to mental health problems due to COVID-19-related disruptions. She suggested the profession be defined as high-risk [13].

On analyzing the pandemic in relation to caregiving by parents and its potential effect on their child's mental health, the COVID-19 pandemic was described by Cohodes EM, et al. [14] as "a universal stressor" that affected all American families, regardless of their socioeconomic status, ethnicity, race, or location [13]. And yet, certain groups are more susceptible than others to these stressors. One population group under analysis, for example, is children with special needs: behavioral and mental health needs. According to Masonbrink AR, et al. [1], 80\% of American children with special needs depend on services that are school-based. This means that pandemic-related closures diminished children's access to meaningful resources, such as learning with adept educators, adapted learning environments, and structured engagement. Older children (young adults) were also not immune to the stresses: an interesting study carried out during the COVID-19 pandemic examined the effects on university students of distance online learning. Lall S, et al. [15] showed that $26 \%$ of students reported a need for faceto-face contact with their peers and expressed negative feelings towards distance learning.

\section{Available Resources Supported Wellbeing}

Compas BE, et al. [16] explored children and adolescents coping with stress and found that positive outcomes depended on the availability of social support resources. Children and adolescents who were exposed to similar amounts of stress managed it differently and more or less successfully depending on the available social resources. Turner $\mathrm{M}$, et al. [17] conducted research on university students and showed that individual resilience can is an essential precursor to wellbeing. An individual develops resilience by drawing positive influences from a variety of resources from different elements of life: home, university, or work circles. Similarly, Turner M, et al. [17] noted that when dealing with a distressing situation, the ability to successfully adapt, manage, and cope with a crisis correlates to the resources the individual has available and his or her immediate environment. Cohodes EM, et al. [14] identified parents as the main support network (source of resiliency) for adolescents and children. Note that this research was carried out prior to the pandemic. Now when taking into 


\section{Mental Health \& Human Resilience International Journal}

consideration the lockdowns and periods of quarantine that families endured during the COVID-19 pandemic, which left children and adolescents cut off from their usual social connections and support circles, parents-if not alreadybecame the main (if not the only) resource remaining for adolescents and children to access.

When preparing a support plan for young individuals with mental health problems, the first step is to identify existing resources. These are often school-based. Kelly $\mathrm{H}$ [4] refers to the Australian Mental Health of Children and Adolescents Report, when writing about the responsibility of schools to offer support for emotional and behavioral problems in youth. Schools play a meaningful role both in identifying mental problems promptly and in attending to students. It is for this reason that Kelly defined schools as "the front-line for dealing with child mental health issues" [12]. Cohodes EM, et al. [14] emphasize the necessity of providing the parents themselves with a support system, given their role as a secondary support circuit for the children, especially during the kind of global events that cause a magnitude of stress to families. Another example of parent/household support that all but disappeared due to COVID-19 related closures is addressed by Masonbrink AR, et al. [1]: in the USA, approximately 6 million children and adolescents from low socio-economic circles normally receive ongoing health support from school-based health centers, and 35 million rely on school nutrition programs. As for older students, taking into consideration the crucial effect that loneliness has on students' wellbeing, Burns D, et al. [2]. Suggested that universities address it as an important phenomenon worth careful treatment. Zhai Y, et al. [18] agreed and suggested that schools consider the stress engendered by the lack of physical interaction and offer online one-on-one talks ("office hours") as a more intimate interaction for students.

After the SARS pandemic, Maunder RG [19] highlighted the importance of acquiring appropriate and measurable treatment for the mental health impact of a health crisis. He suggested that a treatment plan should be adopted simultaneously on multiple therapeutic fronts: psychiatrists and the health care system. The COVID-19 pandemic and the abundant mental health issues which arose in its wake, would be well addressed by policy makers from various fields such as health, therapeutics, and education cooperating to build a holistic recovery and recuperation plan.

\section{Call for Action}

In their article, Masonbrink AR, et al. [1] address the pediatric community and urge them to support (American) national action plans, identifying and answering to children's needs during COVID-19-related closures. They highlight mental health needs alongside physical, nutritional, and educational needs. Psacharopoulos G, et al. [20] warn of an overall educational slide, reflected in decreased student achievements and test scores which may eventually lead to lower earning potentials. COVID-19 related closures led to disconnection from those services which children and adolescents regularly access in educational, social, and emotional fields. This disconnection risks the emergence of a gap in the formation of children's abilities and achievements. Masonbrink AR, et al. [1] emphasized how the young population relies on school-based services for nutritional, physical, and mental health needs. They also identified meaningful sources of emotional support-school counselors, school social workers, and educators-and called on the American government to recognize and provide appropriate professional guidance to these persons who have the potential to positively affect the mental health of school-goers. In a majority of cases, these school-based professionals hold the crucial role of being the first to recognize signs of distress in students.

Kelly $\mathrm{H}$ [5] states that for school systems to develop the capacity to provide effective mental health support to their student population, they need a daily in-school routine alliance with specialists from the public health sector. Mental health support provided by schools should include health promotion, prevention, and early intervention. A detailed plan should be designed such as to compensate for the future estimated educational loss that is expected to result within children overall, and more specifically children in poverty [19].

\section{The International Student Population}

Rajkumar RP [8] analyzed mental health outcomes of the COVID-19 global health crisis; he discovered that several population groups were more vulnerable to mental health problems than others. Two of seven of the groups have an international aspect: Liem A, et al. [21] studied international migrant workers and Zhai $Y$, et al. [22] found stress and symptoms of anxiety in Chinese students studying overseas. Even before the COVID-19 pandemic and its massive emotional effects, Liem A, et al. [21] found that individuals that have a mobile lifestyle which involves regular physical and psychological changes endure intense psychological effects. This is also supported in the Pollock DC, et al. [23] definition of TCK teens as possibly more vulnerable than other teens.

TCKs, by definition, are hosted in a foreign country (i.e. neither they nor their parents are nationals of that country), away from what would be considered their (cultural and linguistic) homeland. Being locked down while a major health crisis rages, detached from their already fragile life circles, social support network, and often newly established daily 


\section{Mental Health \& Human Resilience International Journal}

routine has the potential to increase stress and negatively affect the general wellbeing of the TCKs. These children and adolescents are often observed to suffer from stress and or anxiety, pandemic notwithstanding. A particularly vulnerable group amongst the TCKs are the newcomers-those recently arrived in a third country during the pandemic and its related disruptions. Those children and adolescents were afforded no opportunity to establish or adapt to a 'normal' routine, a social network, or academic practices which were in place in that country (school community) prior to the pandemic. As regards international schools, leaders must recognize the significant and crucial role the school and its support services play in the social and emotional lives of TCKs, who often build their identity in relation to their school's principles and practices. Understanding that international school communities affect the TCK sense of dependence, identity, and attachment is paramount because it also affects their wellbeing and sense of self [24].

\section{Conclusion}

Educators must embrace their function as front-line providers of both academic and emotional support for their students. Based on this understanding, they can advocate for a variety of resources offering emotional support for students. In addressing the socio-emotional effects of COVID-19 related disruptions, schools (whether national or international) should develop thoughtful and detailed mental and social support networks for their students and integration plans for newcomers. Schools can and should work in cooperation with the school community and the locally available human resources. A holistic and effective collaboration is one that combines the available human resources within the school community: school counselors, educators, and the PTO. Expanding the circle, the larger parent community should be recognized as having socio-emotional power that can be built on and developed as an additional resource for schools to tap into. Public decision-makers from the health sector should look to exploit the resources offered through the education system when developing a national mental health support plan for youth and children. As for TCKs, who are not usually considered by the host country's education system, they are left to rely on the International school support system. Therefore, International schools - in cooperation with the parents who spent a year as the almost exclusive source of socio-emotional support for their children - must redouble their efforts to implement social and mental health programs for their students and a COVID-19 related integration plan for newcomers.

\section{References}

1. Masonbrink AR, Hurley E (2020) Advocating for Children During the COVID-19 School Closures. Pediatrics 146(3):
20201440.

2. Burns D, Dagnall N, Holt M (2020) Assessing the Impact of the COVID-19 Pandemic on Student Wellbeing at Universities in the United Kingdom: A Conceptual Analysis. Frontiers in Education.

3. Burki TK (2020) COVID-19: consequences for higher education. Lancet Oncol 21(6): 758.

4. World Health Organization.

5. Kelly H (2021) What are the Most Effective Steps Schools Can Take to Support Teachers' and Leaders' Mental Health and Wellbeing?. The Positive Principal: helping school communities to flourish.

6. Richardson T, Elliott P, Roberts R (2017) Relationship between loneliness and mental health in students. J Public Ment Health 16(2): 48-54.

7. Lyubomirsky S, King L, Diener E (2005) The benefits of frequent positive affect: does happiness lead to success? Psychol Bull 131(6): 803-855.

8. Rajkumar RP (2020) COVID-19 and mental health: A review of the existing literature. Asian J Psychiatr 52: 102066.

9. Fakari FR, Simbar M (2020) Coronavirus Pandemic and Worries during Pregnancy; a Letter to Editor. Arch Acad Emerg Med 8(1): 21.

10. Tsai J, Wilson M (2020) COVID-19: a potential public health problem for homeless populations. Lancet Public Health 5(4): 186-187.

11. Yang Y, Li W, Zhang Q, Zhang L, Cheung T, et al. (2020) Mental health services for older adults in China during the COVID-19 outbreak. Lancet Psychiatry 7(4): 19.

12. Yao H, Chen JH, Xu YF (2020) Rethinking online mental health services in China during the COVID-19 epidemic. Asian J Psychiatr 50: 102015.

13. Kelly H (2021) Should Schools be Responsible for Addressing the Child Mental Health Crisis?. The Positive Principal: helping school communities to flourish.

14. Cohodes EM, McCauley S, Gee DG (2021) Parental Buffering of Stress in the Time of COVID-19: FamilyLevel Factors May Moderate the Association Between Pandemic-Related Stress and Youth Symptomatology. Res Child Adolesc Psychopathol 49(7): 935-948.

15. Lall S, Singh N (2020) COVID-19: unmasking the new face of education. Int J Res Pharm Sci 11(1): 48-53. 


\section{Mental Health \& Human Resilience International Journal}

16. Compas BE, Connor Smith JK, Saltzman H, Thomsen AH, Wadsworth ME (2001) Coping with stress during childhood and adolescence: Problems, progress, and potential in theory and research. Psychol Bull 127(1): 87-127.

17. Turner M, Scott Young CM, Holdsworth S (2017) Promoting wellbeing at university: the role of resilience for students of the built environment. Constr Manag Econ 35(11-12): 707-718.

18. Zhai Y, Du X (2020) Addressing collegiate mental health amid COVID-19 pandemic. Psychiatry Res 288: 113003.

19. Maunder RG (2009) Was SARS a mental health catastrophe?. Gen Hosp Psychiatry 31(4): 316-317.

20. Psacharopoulos G, Patrinos H, Collis V, Vegas E (2020)
The COVID-19 cost of school closures. Brookings, Education plus development.

21. Liem A, Wang C, Wariyanti Y, Latkin CA, Hall BJ (2020) The neglected health of international migrant workers in the COVID-19 epidemic. Lancet Psychiatry 7(4): 20.

22. Zhai Y, Du X (2020) Mental health care for international Chinese students affected by the COVID-19 outbreak. Lancet Psychiatry 7(4): 22.

23. Pollock DC, Van Reken RE (2009) Third Culture Kids: Growing Up among Worlds Rev. Nicholas Brealey Publishing.

24. Lev D (2020) Community and Sense of Place in an International School Context. Studia Doctoralia 11(2): 121-131. 